\title{
Using MAIN in South Africa
}

\section{Daleen Klop}

Stellenbosch University

\section{Monique Visser}

Stellenbosch University

South Africa is a country marked by cultural and linguistic diversity with 11 official languages. The majority of school children do not receive their formal schooling in their home language. There is a need for language assessment tools in education and rehabilitation contexts to distinguish between children with language learning problems and/or SLI, and language delay as a result of limited exposure to the language of learning. The Multilingual Assessment Instrument for Narratives (LITMUS-MAIN) provides clinicians and researchers with an appropriate and culturally relevant tool to assess bilingual children in both languages. So far MAIN has been widely used in AfrikaansEnglish bilingual children. However, translating and adapting MAIN to our other nine official languages to achieve functional and cultural equivalence is more challenging.

\section{Introduction}

This paper describes the use of Language Impairment Testing in Multilingual Settings Multilingual Assessment Instrument for Narratives (LITMUS-MAIN, hereafter MAIN; Gagarina et al., 2012, 2015, 2019) in South Africa by clinicians and researchers and the challenges to adapt MAIN for implementation in our culturally and linguistically diverse context.

\section{$2 \quad$ Linguistic diversity in South Africa}

South Africa is one of the most diverse societies in the world. Multilingualism and cultural diversity are valued and celebrated features of the South African society. In acknowledgement of the importance of our cultural and linguistic diversity, the 11 main languages spoken in South Africa, namely isiZulu, isiXhosa, Sepedi, Setswana, Sesotho, Xitsonga, siSwati, Tshivenda, 
isiNdebele, English and Afrikaans are all recognised as official languages in our Constitution. Afrikaans originated in South Africa and is a West Germanic language derived from Dutch. The official African languages are Bantu languages and belong to four language families namely Nguni (isiZulu, isiXhosa, isiNdebele and siSwati), Sotho-Tswana (Sepedi, Setswana, Sesotho), Xitsonga and Tshivenda. Besides these official languages, many other Southern African Bantu languages such as Siphuthi, Khilobedu, Sindebele, Shona, Shangaan and Chichewa are also spoken. Furthermore, South Africa is home to migrants from African countries where Portuguese and French are spoken. The most common languages used as home languages in South Africa are IsiZulu (23\%), followed by isiXhosa (16\%) and Afrikaans (14\%). (Statistics South Africa, 2016). Although only $10 \%$ of the population speaks English as a home language, it is the prominent political and educational language in South Africa and the language of learning for most school children. Unfortunately, many children start their school careers with limited proficiency in their school language and this, together with poor academic and parental support, often have detrimental effects on their literacy development.

\section{MAIN in South Africa}

There is a dire need in South Africa for language assessment instruments and protocols that are culturally appropriate and linguistically valid. Speech-language pathologists (SLPs), educators and researchers are often confronted with the challenge to disentangle the effects of bilingualism from specific language impairment (SLI) and language delay. Many children in South Africa receive their formal schooling in their second or even third language that may differ substantially from their home language. SLPs and educators are therefore often required to distinguish between children with language learning problems and/or SLI, and language delay as a result of limited exposure to the school language. The majority of SLPs in South Africa are from Afrikaans and English home language backgrounds with limited or no proficiency in the other official languages. To exacerbate this, most SLPs have only access to English standardised language assessment tools that were normed on populations in the USA and UK. Many SLPs use translated versions of these tests that have not been standardised on the South African population (Southwood \& Van Dulm, 2015). There is therefore a great need for protocols that could assess bilingual children in both their languages, while taking into consideration their experiential, linguistic and cultural backgrounds.

The assessment of narratives is deemed to be an ecologically valid way to investigate communicative competence and is often regarded as more sensitive and less biased than normreferenced assessment tools that target discrete aspects of language in some populations (Manolitsi \& Botting, 2011). The Multilingual Assessment Instrument for Narratives (MAIN) (Gagarina et al., 2019) was specifically developed to assess the narrative production and comprehension of bilingual children in a way that is culturally fair and unbiased. The developers strived to develop pictorial stimuli that are accessible to children from diverse cultural, linguistic and socio-economic backgrounds and the pilot studies in a variety of languages across the world confirmed the cultural robustness of this instrument (Gagarina et 
al., 2015). The adaptation and translation of MAIN to all official languages in South Africa will therefore provide clinicians and researchers with much-needed instruments to assess language in our population.

Adapting MAIN for use in South Africa is, however, not without challenges. The rich oral tradition and practices associated with African cultures provide children with language and learning experiences that are often very different from typical Western contexts. It is therefore not a straightforward process to adapt and translate MAIN from English or Afrikaans to an African language, for instance isiZulu. Measures to ensure linguistic equivalence through processes such as back-translation or double translation may still not result in functional and cultural equivalence. Functional equivalence refers to the adaptation of protocols so that they represent concepts in ways that are familiar to participants from different linguistic or cultural groups. For instance, functional equivalence would include measures to ensure that the isiZulu adaptation of MAIN from English still examines the same constructs such as goal structures in both languages. Cultural equivalence aims to ensure that members from different cultural and linguistic groups view and interpret the instructions and underlying meaning of constructs in the same way (Pena, 2007). Recent adaptions to the MAIN picture stimuli, such as adjusting the boy's skin colour and replacing the sausages in the boy's bag with chicken legs, has improved the content validity and cultural appropriateness of MAIN for use in the South African context. MAIN has so far been translated to isiXhosa and Tshivenda, but more work is needed to ensure functional and cultural equivalence for these versions. Following the procedures stipulated by the International Test Commission Guidelines (Bartram et al., 2018), research projects are underway to consult with cultural insiders in isiXhosa and Tshivenda populations to investigate the appropriateness and validity of MAIN in their contexts.

\section{$4 \quad$ Clinical applications}

MAIN has been used in the Speech-Language Therapy Clinic at Stellenbosch University not only for children with language problems, but also for the assessment of children with speech fluency disorders such as stuttering and cluttering. Diagnostic assessment procedures for fluency disorders usually include in-depth analyses of speech samples to determine the nature and frequency of the disfluent speech behaviour. It is important to obtain a variety of samples because speech fluency is influenced by the linguistic and speech-motor planning demands of different speech and language tasks (Guitar, 2014). Furthermore, it is recommended that bilingual children with stuttering are assessed in both languages to determine and compare the nature and extent of their speech disfluencies in both languages, and to assess the impact of language proficiency in both languages on their stuttering (Shenker, 2011). MAIN provides clinicians with an appropriate tool to obtain speech samples at discourse level for fluency analyses, and at the same time clinical information about their narrative production and comprehension. 


\section{Conclusion}

At present, the Afrikaans and English versions of MAIN are used in South Africa in monolingual and bilingual populations in both research and clinical settings. The Afrikaans adaptation has been piloted in a variety of contexts and populations and found to be an effective tool to assess narrative production and comprehension in Afrikaans-English bilingual populations. However, translating and adapting MAIN to our other nine official languages is more challenging and several projects are underway to create functional and cultural equivalent versions of MAIN in these languages.

\section{$6 \quad$ References}

Bartram, D., Berberoglu, G., Grégoire, J., Hambleton, R., Muniz, J., \& van de Vijver, F. (2018). ITC Guidelines for Translating and Adapting Tests (Second Edition). International Journal of Testing, 18(2), 101-134.

Gagarina, N., Klop, D., Kunnari, S., Tantele, K., Välimaa, T., Balčiūnienė, I., Bohnacker, U., \& Walters, J. (2012). Multilingual Assessment Instrument for Narratives (MAIN). ZAS Papers in Linguistics, 56.

Gagarina, N., Klop, D., Kunnari, S., Tantele, K., Välimaa, T., Balčiūnienė, I., Bohnacker, U., \& Walters, J. (2015). Assessment of narrative abilities in bilingual children. In S. Armon-Lotem, J. de Jong, \& N. Meir (Eds.), Assessing multilingual children: Disentangling bilingualism from language impairment (pp. 243-269). Bristol: Multilingual Matters.

Gagarina, N., Klop, D., Kunnari, S., Tantele, K., Välimaa, T., Balčiūnienė, I., Bohnacker, U., \& Walters, J. (2019). MAIN: Multilingual Assessment Instrument for Narratives - Revised. ZAS Papers in Linguistics, 63.

Guitar, B. (2019). Stuttering: An Integrated Approach to its Nature and Treatment (5th ed). Baltimore, MD; Lippincott, Williams \& Wilkins.

Manolitsi, M., \& Botting, N. (2011). Language abilities in children with autism and language impairment: Using narrative as an additional source of clinical information. Child Language Teaching and Therapy, 27(1), $39-55$.

Peña, E. D. (2007). Lost in translation: Methodological considerations in cross-cultural research. Child Development, 78(4), 1255-1264.

Shenker, R. C. (2011). Multilingual children who stutter: Clinical issues. Journal of Fluency Disorders, 36(3), $186-193$.

Southwood, F., \& Van Dulm, O. (2015). The challenge of linguistic and cultural diversity: Does length of experience affect South African speech-language therapists' management of children with language impairment? South African Journal of Communication Disorders, 62(1), 1-14.

Statistics South Africa. (2016). Census 2011: Census in brief. Retrieved from http://www.statssa.gov.za/Census2011/Products/Census_2011_Census_in_brief.pdf 